\title{
Computer Science Tools for Manual Editing of Computed Tomographic Images: Impact on the Quality of 3D Printed Models
}

\author{
Jean H. D. Fasel1* Jörg Beinemann², Karl Schaller ${ }^{3}$, Heinz-Otto Peitgen4 \\ ${ }^{1}$ Clinical Anatomy Research Group, Department of Cellular Physiology and Metabolism, University Medical \\ Center, Geneva, Switzerland \\ ${ }^{2}$ Hightech Research Center of Cranio-Maxillofacial Surgery, University of Basel, Basel, Switzerland \\ ${ }^{3}$ Department of Neurosurgery, University Hospitals, Geneva, Switzerland \\ ${ }^{4}$ Department of Mathematical Sciences, Florida Atlantic University, Boca Raton, USA \\ Email: ${ }^{*}$ jean.fasel@unige.ch
}

Received 16 August 2014; revised 15 September 2014; accepted 10 October 2014

Copyright (C) 2014 by authors and Scientific Research Publishing Inc.

This work is licensed under the Creative Commons Attribution International License (CC BY).

http://creativecommons.org/licenses/by/4.0/

(c) (7) Open Access

\section{Abstract}

Background: Three-dimensional printing (3DP) technologies are a trendsetting topic, also in the field of surgery. Preoperative planning for maxillofacial and neurological surgery, for instance, increasingly involves skull models obtained by 3DP. However, the cranial replicas currently used in this context have been shown to not reproduce the exact anatomy of the individual patient undergoing surgery. Objective: The present study aimed at investigating the extent to which manual editing, using current computer software tools, can improve skull models derived from medical images. Methods: Skull computed tomography (CT) was obtained on three cadavers and sent to three institutions that provide preoperative 3DP services. Each institute independently performed 3D reconstructions, including routine manual editing, and subsequently produced the replicas. The models were then qualitatively compared with the respective original skull. For quantitative comparison surface scans of particular regions of interest were made and the deviations assessed using 3-matic software (Materialise, Leuven, Belgium). Results: Routine manual editing of CT images resulted in replicas that were clear improvements over automatically generated reconstructions. This was particularly the case for teeth artefacts and thin-walled entities (e.g. paranasal sinuses). Conversely, however, many anatomical structures remained incorrectly rendered (e.g. orbitae, pterygoid processes, and sella turcica). Extraosseous calcifications had regularly not been removed. After extensive manual editing, however, replicas were able to provide largely submillimeter accuracy (mean deviation $0.2496 \mathrm{~mm}$; standard deviation $\mathbf{\pm 0 . 2 2 7 6} \mathrm{mm}$ ). Conclusions: This study confirms that manual editing with current computer science tools does improve the quality of CT-based 3D printed skull models. But, it also demonstrates that a number

*Corresponding author. 
of structures remain largely incorrectly rendered when edited in the presently used surgical framework. Conversely, it shows that highly accurate replicas are feasible, provided that extensive manual editing is performed.

\section{Keywords}

\section{Preoperative Replicas, 3D Printing, Skull, Models, Accuracy}

\section{Introduction}

Additive manufacturing technologies (such as stereolithography and selective laser sintering) refer to processes in which virtual mathematical 3D models are used to build up real physical objects through joining materials layer by layer. They can be thought of as the opposite of subtractive methods (such as drilling and milling) and are generally considered to hold huge potential for innovations, including medical applications [1] [2]. For surgery, for instance, models of human organs obtained by using three-dimensional printing (3DP) are being increasingly used for preoperative planning [3] [4] and postgraduate education [5] [6].

As far as maxillofacial and neurological surgeries are concerned, the quality of the skull replicas currently obtained is generally accepted by surgeons, even though recent investigations have shown that these models do not reliably render the detailed anatomy of the individual patient [7] [8], confirming reports from the 1990s [9] [10].

In contrast to the said conventional opinion amongst clinicians, this paper presumes that, because of the strong trend towards minimally invasive and personalized surgery, it seems likely that more accurate skull models than those currently used will be needed. That is why the present study aimed to investigate the extent to which manual editing - using current computer software tools — can improve skull models derived from medical imaging, and thus integrate patient-specific data for procedural planning and educational purposes in surgery.

\section{Materials and Methods}

In accordance with the Swiss legislation that allows research on donated bodies, the craniofacial bony complexes of three cadavers donated to the Geneva anatomy department were acquired with computed tomography (CT) using routine clinical parameters (Somatom Sensation 64, Siemens, Erlangen, Germany), The DICOM data were then sent to three independent institutes with 3DP facilities. Each institute was asked to perform routine 3D reconstructions, including manual editing, and to produce a physical model of each skull for preoperative planning purposes. Detailed visual comparisons of the replicas were then carried out against the original skulls—region by region, structure by structure-according to the current international anatomical list [11]. For a quantitative analysis the surfaces of a replica and the respective original skull were scanned using an ATOS triple scan (GOM GmbH, Braunschweig, Germany). Deviations between the real and the manufactured skull were assessed using the Mimics Innovation Suite Research 3-matic software (Version 9.0; Materialise, Leuven, Belgium).

\section{Results}

The topographic and systematic comparison of the replicas against their respective original skulls confirmed that routine manual editing of the CT images, using the above algorithms, yields unambiguous improvements in the models compared to reconstructions generated purely automatically. The most striking example concerned teeth artefacts (Figure 1).

However, even after manual editing had been performed under a routine surgical context (such as preoperative planning purposes), many flaws in the replica remained unchanged. Extraosseous calcifications had frequently not been removed. For example, Figure 2(A) and Figure 2(B) show the region of a sella turcica in the case of advanced internal carotid artery atheromatosis, obstructing the view of the parasellar area in the replica. In Figure 2(D) the model shows artefactual defects in the base of the medial plate of the pterygoid process.

\section{Discussion}

This study's results confirm that routine manual editing of computed tomographic skull images, using currently 

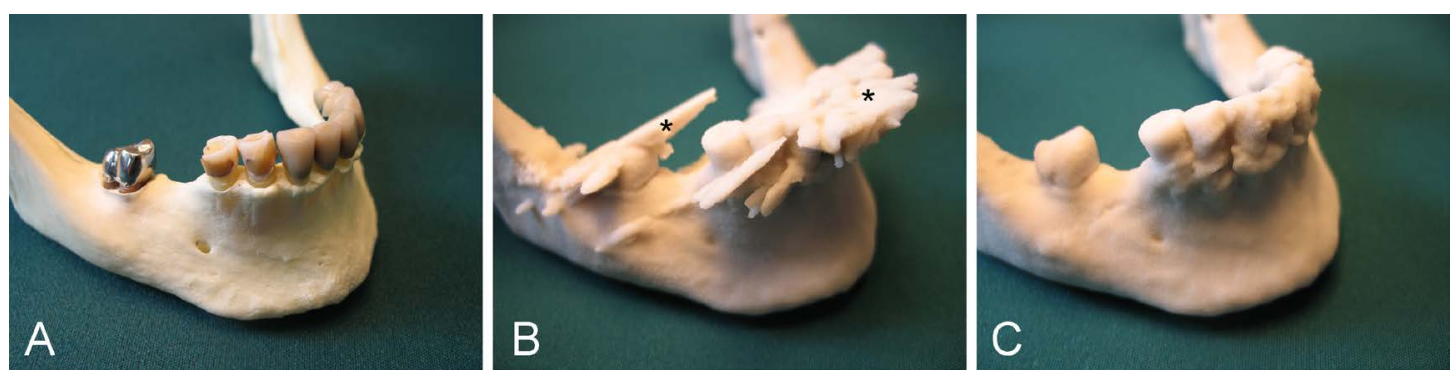

Figure 1. Examples of 3D printed models of a mandible compared to the original specimen. (A) The original mandible. (B) The replica obtained via automatic segmentation of the CT images displays typical radiate teeth artefacts (indicated by asterisks: ${ }^{*}$ ). (C) A marked reduction of these artefacts after routine manual editing. The Figure documents that $3 \mathrm{D}$ printed models can be improved by manual editing of the CT images.
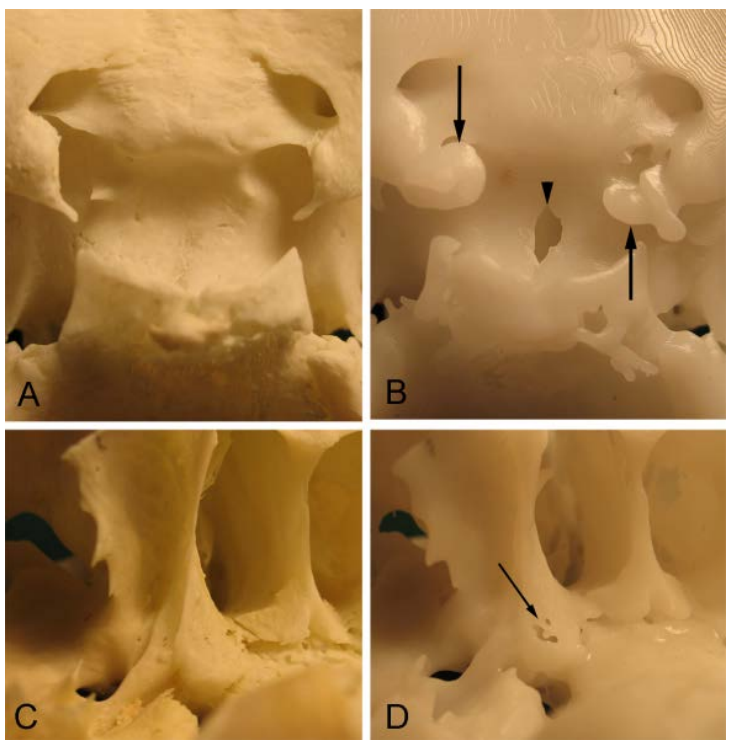

Figure 2. Examples of skull regions that are consistently incorrectly replicated despite routine manual editing. (A) and (C) show the regions in the original skulls. (B) and (D) are the corresponding areas in the 3D printed replicas.(A) and (B) show the sella turcica. Note the calcified internal carotid arteries (arrows) in the replica falsely mimicking a non-existent caroticoclinoid ring and hiding the middle clinoid processes. Further, note the artefactual foramen (arrowhead) in the bottom of the sella, which may suggest the presence of a craniopharyngeal canal. (C) and (D) show the base of the right medial pterygoid lamina. Note the presence of artefactual foramina (arrow) in the replica-not to be mistaken for accessory openings around the pterygoid (vidian) canal. The Figure illustrates that routine manual editing can fail to yield 3D printed models that reliably reflect the detailed osseous anatomy of the individual patient's skull.

available computer software tools, helps to improve the quality of 3D printed models as compared to those obtained using automated procedures (Figure 1). However, the investigation also shows that manual editing-as is routinely performed in a clinical context (particularly for preoperative planning purposes) - is far from guaranteeing a true reproduction of the specific skull anatomy of the patient.

This finding is of immediate clinical relevance: A wrongly mimicked caroticoclinoid foramen (Figure 2(B)) could have an impact on directing transsphenoidal endoscopic approaches, for example [12]. Artefactual foramina could be mistaken either for normal variants or for pathological conditions such as a craniopharyngeal canal [13] or accessory bony openings for neurovascular bundles around the pterygoid (vidian) canal (Figure 2(D)) — this canal being a useful landmark for a skull base neurosurgeon [14] carrying out a vidian neurectomy, for instance [15].

In order to clarify whether, or to what extent, a much more detailed manual editing than is routinely done in a surgical context could reduce flaws, certain skull regions particularly prone to errors were further edited on one of the skulls. Figure 3 illustrates the results for the sella turcica. A further marked qualitative improvement was 

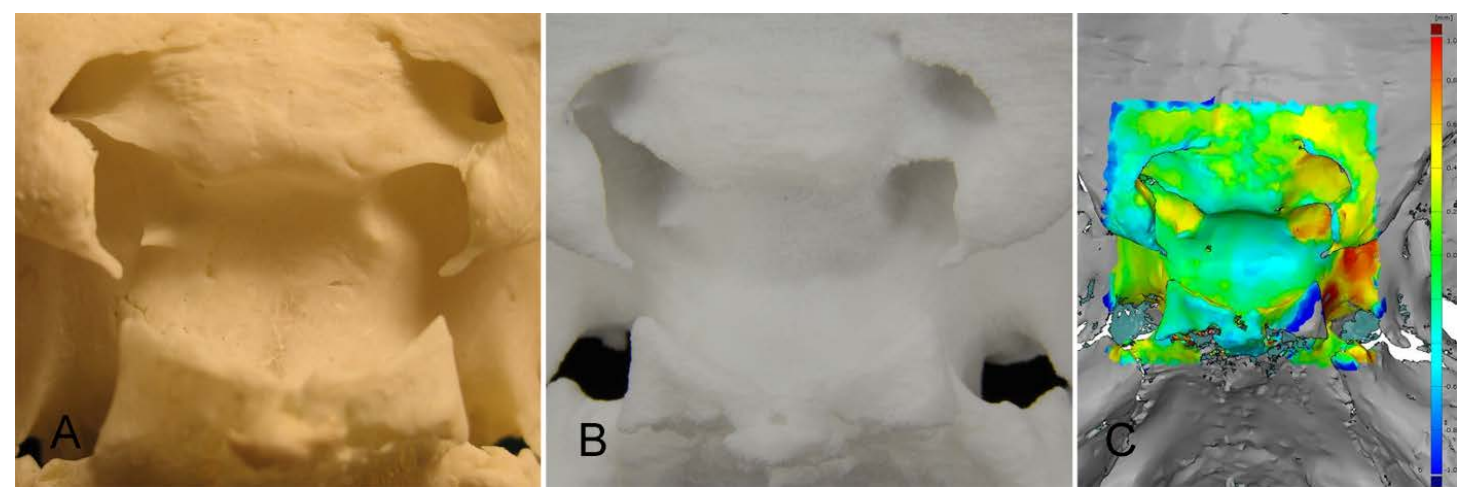

Figure 3. The impact of extensive manual editing of CT images on the quality of replicas. (A) The sella in the original skull. (B) The replica following extensive manual editing. (C) The quantitative comparison of both. Note the excellent qualitative and quantitative match between the 3D printed model and the original.

obtained. The errors observed after routine manual editing (Figure 2(B)) were fully eliminated, the middle clinoid processes and their asymmetry became clearly visible, and the caroticoclinoid foramen was gone. The pseudo-craniopharyngeal canal also disappeared (Figure 3(B)).

Quantitatively, the conformity of the model was also excellent. Figure 3(C) shows the corresponding color-coded deviation graph. The median deviation was $0.1836 \mathrm{~mm}$ (range $0.0000 \mathrm{~mm}$ to $1.4368 \mathrm{~mm}$ ); the mean deviation amounted to $0.2496 \mathrm{~mm}$ (standard deviation $\pm 0.2276 \mathrm{~mm}$ ). However, it has to be noted that the extensive manual editing necessary for the only small region of interest illustrated in Figure 3 took ten hours to carry out. This is obviously beyond the scope of any standard clinical context.

Highly accurate replicas (with largely submillimeter accuracy) are, therefore, obtainable using current computer software tools for manual editing. However, providing anatomical models of such quality is only possible with great expenditure of time and money and with provision of high professional anatomical standards.

\section{Conclusion}

This investigation confirms that manual editing using current computer software tools can improve the quality of 3D printed physical replicas based on CT images. However, it also demonstrates that a number of structures are still largely incorrectly rendered when edited following routine surgical frameworks. In view of the increasing demand for minimally invasive and personalized surgical procedures, it seems likely that surgeons will need more accurate skull models than those currently used. This study shows that highly accurate reproductions, both qualitatively and quantitatively, are indeed feasible if extensive manual editing is performed.

\section{References}

[1] Esses, S.J., Berman, P., Bloom, A.I., et al. (2011) Clinical Applications of Physical 3D Models Derived from MDCT Data and Created by Rapid Prototyping. American Journal of Roentgenology, 196, W683-W688. http://dx.doi.org/10.2214/AJR.10.5681

[2] Rengier, F., Mehndiratta, A., von Tengg-Kobligk, H., et al. (2010) 3D Printing Based on Imaging Data: Review of Medical Applications. International Journal of Computer Assisted Radiology and Surgery, 5, 335-341. http://dx.doi.org/10.1007/s11548-010-0476-x

[3] Spottiswoode, B.S., van den Heever, D.J., Chang, Y., et al. (2013) Preoperative Three-Dimensional Model Creation of Magnetic Resonance Brain Images as a Tool to Assist Neurosurgical Planning. Stereotactic and Functional Neurosurgery, 91, 162-169. http://dx.doi.org/10.1159/000345264

[4] Zein, N.N., Hanouneh, I.A., Bishop, P.D., et al. (2013) Three-Dimensional Print of a Liver for Preoperative Planning in Living Donor Liver Transplantation. Liver Transplantation, 19, 1304-1310. http://dx.doi.org/10.1002/lt.23729

[5] Waran, V., Narayanan, V., Karuppiah, R., et al. (2014) Injecting Realism in Surgical Training-Initial Simulation Experience with Custom 3D Models. Journal of Surgical Education, 71, 193-197. http://dx.doi.org/10.1016/j.jsurg.2013.08.010

[6] Watson, R.A. (2014) A Low-Cost Surgical Application of Additive Fabrication. Journal of Surgical Education, 71, 1417. http://dx.doi.org/10.1016/j.jsurg.2013.10.012 
[7] Fasel, J.H.D., Beinemann, J., Schaller, K., et al. (2013) A Critical Inventory of Preoperative Skull Replicas. Annals of The Royal College of Surgeons of England, 95, 401-404. http://dx.doi.org/10.1308/003588413X13629960046994

[8] Huotilainen, E., Jaanimets, R., Valásek, J., et al. (2014) Inaccuracies in Additive Manufactured Medical Skull Models Caused by the DICOM to STL Conversion Process. Journal of Craniomaxillofacial Surgery, 42, e259-e265. http://dx.doi.org/10.1016/j.jcms.2013.10.001

[9] Sader, R., Zeilhofer, H.F., Kliegis, U., et al. (1997) Precision of 3D-Assisted Surgical Planning with Rapid Prototype Techniques. Mund-, Kiefer- und Gesichtschirurgie, 1, S61-S64.

[10] Lill, W., Solar, P., Ulm, C., et al. (1992) Reproducibility of Three-Dimensional CT-Assisted Model Production in the Maxillofacial Area. British Journal of Oral and Maxillofacial Surgery, 30, 233-236. http://dx.doi.org/10.1016/0266-4356(92)90265-K

[11] Federative Committee on Anatomical Terminology (FCAT) (1998) Terminologia Anatomica. Thieme, Stuttgart.

[12] Peris-Celda, M., Kucukyuruk, B., Monroy-Sosa, A., et al. (2013) The Recesses for the Sellar Wall of the Sphenoid Sinus and Their Intracranial Relationships. Neurosurgery, 73, ons117-ons131. http://dx.doi.org/10.1227/NEU.0000000000000184

[13] Abele, T.A., Salzman, K.L., Harnsberger, H.R., et al. (2014) Craniopharyngeal Canal and Its Spectrum of Pathology. American Journal of Neuroradiology (AJNR), 35, 772-777. http://dx.doi.org/10.3174/ajnr.A3745

[14] Kassam, A.B., Vescan, A.D., Carrau, R.L., et al. (2008) Expanded Endonasal Approach: Vidian Canal as a Landmark to the Petrous Internal Carotid Artery. Journal of Neurosurgery, 108, 177-183. http://dx.doi.org/10.3171/JNS/2008/108/01/0177

[15] Liu, S.C., Wang, H.W., Kao, H.L., et al. (2013) Three-Dimensional Bone CT Reconstruction Anatomy of the Vidian Canal. Rhinology, 51, 306-314. 
Scientific Research Publishing (SCIRP) is one of the largest Open Access journal publishers. It is currently publishing more than 200 open access, online, peer-reviewed journals covering a wide range of academic disciplines. SCIRP serves the worldwide academic communities and contributes to the progress and application of science with its publication.

Other selected journals from SCIRP are listed as below. Submit your manuscript to us via either submit@scirp.org or Online Submission Portal.
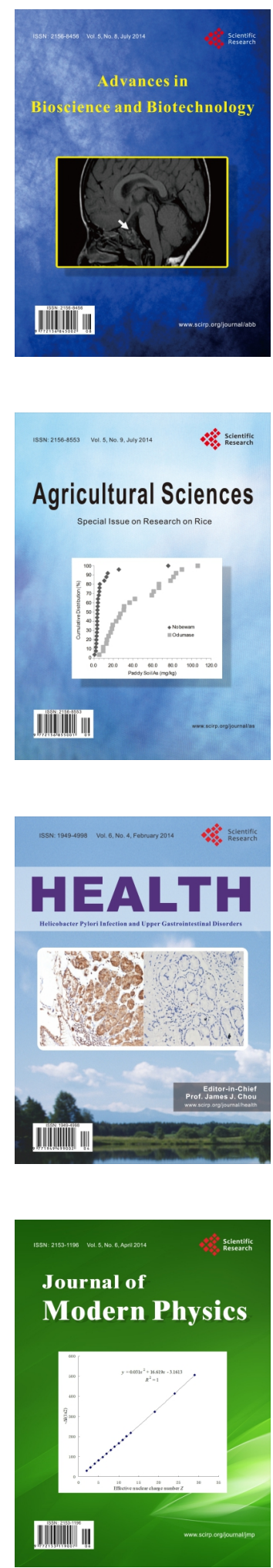
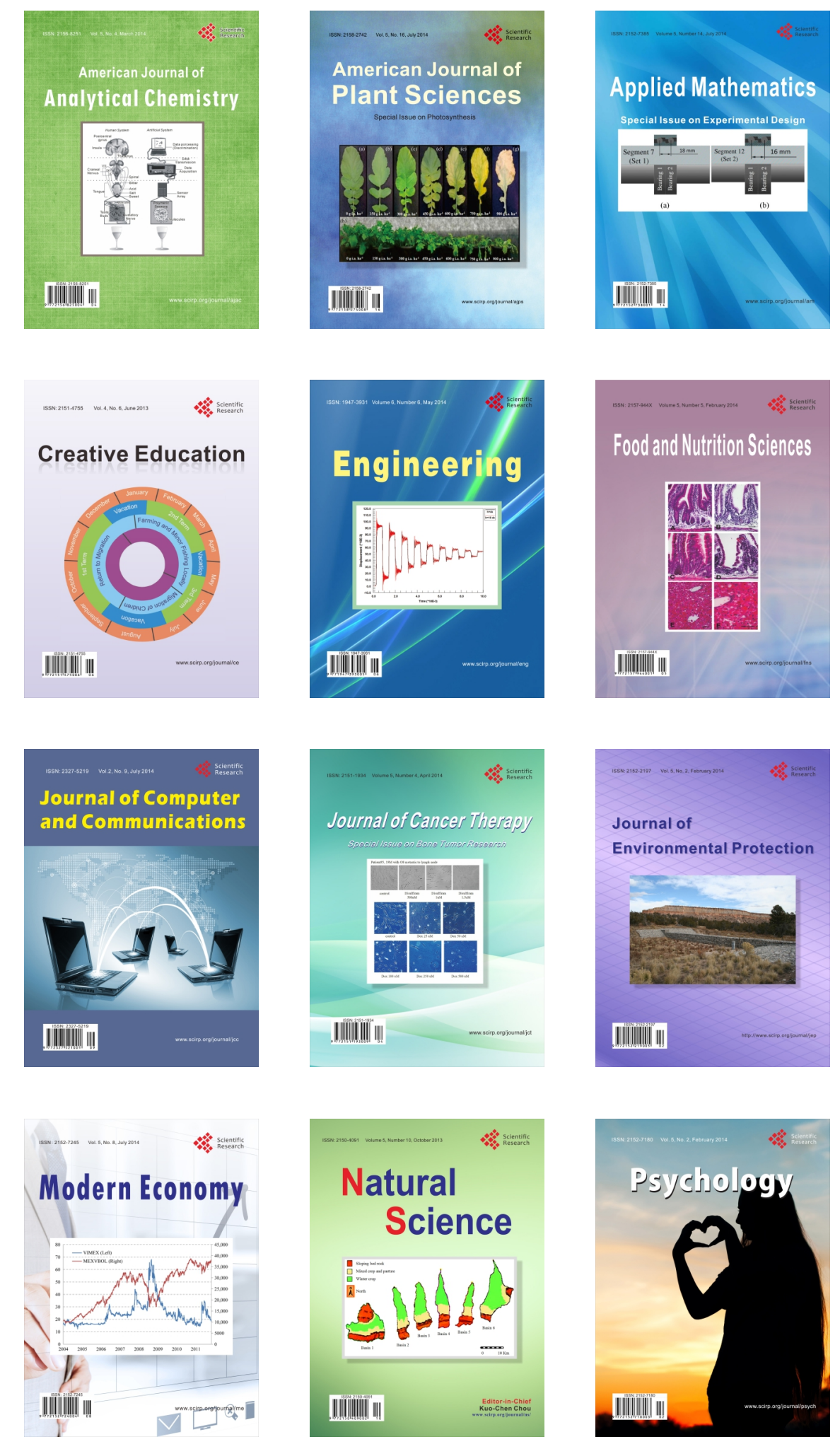